\title{
Estimulación reproductiva en burras en posparto, bajo pastoreo controlado en región tropical seca
}

\author{
Aguirre Riofrio, L. ${ }^{@}$ y Soto, S.C. ${ }^{2}$ \\ 'Centro de Biotecnología Reproductiva Animal. Universidad Nacional de Loja. Ecuador. \\ ${ }^{2}$ Estación Experimental Punzara. Facultad de Ciencias Veterinarias. Universidad Nacional de Loja. Ecuador.
}

\section{Palabras clave adicionales}

Equus asinus.

Estro.

Preñez.

Protocolos de sincronización.

\section{ADDITIONAL KEYWORDS \\ Equus asinus. \\ Gestation. \\ Oestrus. \\ Synchronization protocols.}

\section{INFORMACIÓN}

Cronología del artículo.

Recibido/Received: 25.4.2014

Aceptado/Accepted: 11.11.2014

On-line: 16.3.2015

Correspondencia a los autores/Contact e-mail:

leninaguirrer@yahoo.es

\section{RESUMEN}

Este trabajo fue desarrollado para investigar el comportamiento reproductivo a nivel del trópico seco de las burras Criollas en anestro posparto. Veinticuatro hembras, mantenidas en pastoreo controlado fueron sometidas a tres tratamientos de sincronización: T1: día 0 implante auricular de $3 \mathrm{mg}$ norgestomet (Crestar ${ }^{\circledR}$, Intervet Inc.) +50 mg P4/IM (Progesterona $\AA$, Erma), día 12 retiro del implante y $0,25 \mathrm{mg} / \mathrm{IM}$ de cloprostenol sódico (Lutaprost $₫$, Agrovetmarket S.A.); T2: día 0 se aplicó $250 \mu$ g de gonadorelin, GnRH /IM (Fertagy|®, Intervet Inc.) y el día 12 vía IM 0,25 mg cloprostenol sódico (Lutaprost ${ }^{\circledR}$ ); T3: día 0 dispositivo vaginal 0,5 g P4 (DIB $\circledast$, Sintex), día 12 se retiró dispositivo + 0,25 mg/IM de cloprostenol sódico (Lutaprost $\mathbb{B}$ ). Los incrementos de progesterona en sangre (P4) en los tratamientos hormonales, el inicio, duración y comportamiento del celo sincronizado y la eficacia de los protocolos mediante el diagnóstico ecográfico de gestación al día 50 poscubrición con monta directa el día 2 y 3 de iniciado el celo, fueron evaluados. Los tres protocolos analizados provocaron un incremento en los niveles de P4 de $16,6 \pm 8,8 \mathrm{ng} / \mathrm{mL}$, no existiendo diferencia entre ellos; todas las burras presentaron un celo manifiesto que se inició a los $3,3 \pm 0,4$ días en los protocolos con implantes auriculares y dispositivos vaginales y a los $3,9 \pm 0,5$ días en el protocolo usando $\mathrm{GnRH}(p<0,05)$. La conducta estral, medida por la presencia de todos, o una parte, de los síntomas típicos en esta especie no fue uniforme, presentando un comportamiento óptimo el $46 \%$ de los animales, muy bueno el $37 \%$ y el $17 \%$ restante un celo bueno, destacándose en este sentido el protocolo con implante auricular. La duración del celo se relacionó con el comportamiento, así, celos óptimos y muy buenos tuvieron una duración de 4 días y celos buenos, de 5 días. El porcentaje de concepción fue de $100 \%$ en las burras con implantes auriculares, del $75 \%$ en el protocolo de $\mathrm{GnRH}$ y del 37,5\% en las de dispositivo vaginal $(p<0,05)$. En este último tratamiento se observó catarro vaginal en todas las burras al momento de la extracción del dispositivo, lo que, al parecer, afecta notoriamente al proceso de concepción por monta natural.

\section{Postpartum reproductive stimulation of jennies under controlled grazing in dry tropical region}

\section{SUMMARY}

This work was developed to investigate the Criollo donkey's reproductive behavior in postpartum anoestrus at the dry tropic. For this investigation, twenty four females handled under controlled grazing, were subjected to three synchronization treatments: $T 1$ : day 0 , ear implant of $3 \mathrm{mg}$ norgestomet (Crestar $\AA$, Intervet Inc.) +50 mg P4/IM (Progesterona $®$, Erma), day 12th, implant removal and administration of $0.25 \mathrm{mg} / \mathrm{IM}$ sodium cloprostenol (Lutaprost $\Theta$, Agrovetmarket S.A.); T2: day 0, was applied $250 \mu \mathrm{g}$ of gonadorelin, GnRH/IM (Fertagy|®, Intervet Inc.) and, day 12 th, IM administration of $0.25 \mathrm{mg}$ sodium cloprostenol (Lutaprost $\Theta)$; T3: day 0, placement of vaginal device, $0.5 \mathrm{~g} \mathrm{P4}(\mathrm{DIB} \circledast$, Sintex), day 12 th, device removal + administration of $0.25 \mathrm{mg} / \mathrm{IM}$ of sodium cloprostenol (Lutaprost $₫$ ). The three protocols analyzed, resulted in an increase in the level of P4 of $16.6 \pm 8.8 \mathrm{ng} / \mathrm{mL}$, without any difference between protocols; the female donkeys exposed to the protocols with ear implants and vaginal devices, manifested an estrus that began at $3.3 \pm 0.4$ days. On the other hand, with the protocol using $\mathrm{GnRH}$, the estrus was manifested at $3.9 \pm 0.5$ days $(p<0.05)$. When the presence of all or part of the typical symptoms of this species was measured, the estrous was not uniform; of $46 \%$ of animals, showed an optimal behavior, in $37 \%$ of animals a very good pattern was registered and in the remaining $17 \%$ oestrus was just good, excelling in this variable the ear implant protocol. The duration of estrus was associated with the estrus pattern, so, optimal and very good estrus, lasted four days and good estrus lasted five days. The conception rate was $100 \%$ in the jennies with ear implants, $75 \%$ in the protocol of $\mathrm{GnRH}$ and $37.5 \%$ in the vaginal device $(p<0.05)$. In this treatment the presence of vaginal catarrh was detected, when the devices were removed in all jennies, this problem apparently affects the process of conception by natural mating. 


\section{INTRODUCCIÓN}

En los caballos se presenta una estacionalidad anovulatoria que está influenciada fundamentalmente por el ambiente (Pycock, 2001), similar situación aunque en menor grado se ha encontrado en los asnos (Blanchard et al., 1999; Galisteo y Perez-Marin, 2010), análisis que se han realizado a nivel de zonas templadas; pero en el trópico seco, al factor clima se le debe añadir el sistema de pastoreo controlado en áreas de escasa vegetación, pues sus propietarios les brindan muy poca atención lo que afecta la condición corporal posparto, que sumado a la presencia de la cría al pie provoca un periodo de anestro durante la época seca.

En la región tropical seca del continente americano y de la zona del Pacífico, son pocos o inexistentes los estudios que se hayan realizado y publicado sobre el comportamiento reproductivo de la hembra asnal (Equus asinus), más aún considerando que vienen a representar una población genéticamente diferente a la proveniente de la parte americana de la costa atlántica, Europa y África (Jordana et al., 2012). El material investigativo realizado en la parte reproductiva en esta especie, se refiere a la conducta reproductiva y ciclo estral (Blanchard et al., 1999; Taberner et al., 2008), esquema hormonal (Meira et al., 1995), dinámica folicular (Dadarwal et al., 2004; Lemma et al., 2006) y dentro de la sincronización de celos, empleando prostaglandinas (Carluccio et al., 2008; Miro et al., 2010) y progestágenos por vía vaginal con escaso éxito (Miro et al., 2011); no disponiéndose de información referente a la utilización de progestágenos por otras vías y de la GnRH como inductores de celo.

El objetivo del presente trabajo fue evaluar el comportamiento reproductivo de la burra Criolla en el periodo del posparto, sometida a protocolos hormonales con el uso de progestágenos y GnRH, determinar los niveles de $\mathrm{P} 4$ al inicio y final de los tratamientos, la conducta sexual de las hembras inducidas y la eficacia de los mismos por medio del diagnóstico de preñez, permitiendo con ello disponer de herramientas para el mejoramiento de la eficiencia reproductiva en esta especie animal de la cual se tiene poca información técnica al respecto.

\section{MATERIAL Y MÉTODOS}

El trabajo se realizó en el sector Nambacola, provincia de Loja, al sur del Ecuador (S4 ${ }^{\circ} 0^{\prime} / \mathrm{S}^{\circ}{ }^{\circ} 1^{\prime}$; W79 ${ }^{\circ}$ $30^{\prime} / \mathrm{W} 79^{\circ} 05^{\prime}$ ) a $920 \mathrm{msnm}$, con precipitación anual de $750 \mathrm{~mm}$ y temperatura media de $22^{\circ} \mathrm{C}$; se desarrolló entre julio y octubre del 2013, época de escasez de pasto, empleando 24 burras Criollas entre 3 y 6 años de edad con cría al pie y en anestro posparto mayor a 2 meses y tres burros machos del lugar, de comprobada fertilidad; los días 0 y 12, considerados como de inicio y finalización de los tratamientos se recolectaron muestras de sangre sin anticoagulante para análisis de niveles de P4 en suero sanguíneo, por medio de enzimoinmunoanálisis ELISA, empleando para ello el kit de diagnóstico comercial Progestone ${ }^{\circledR}$ (Human. Germany).
Se asignaron en forma aleatoria 8 individuos a cada tratamiento, vale señalar que para una mejor evaluación, se iniciaron cada uno con un intervalo de 10 días, el tratamiento T1 consistió: día 0 implante auricular de $3 \mathrm{mg}$ norgestomet (Crestar ${ }^{\circledR}$, Intervet Inc.) +50 mg P4/ IM (Progesterona ${ }^{\circledR}$, Erma), día12 retiro del implante y 0,25 mg/IM de cloprostenol sódico (Lutaprost $\AA$, Agrovet-market S.A.); T2: día 0 se aplicó 250 g de gonadorelin/IM GnRH (Fertagyl ${ }^{\circledR}$, Intervet Inc.) y el día 12 vía IM 0,25 mg cloprostenol sódico (Lutaprost巴); T3: día 0 dispositivo vaginal 0,5 g P4 (DIB $\AA$, Sintex), día 12 se retiró dispositivo $+0,25 \mathrm{mg} / \mathrm{IM}$ de cloprostenol sódico (Lutaprost $\AA$ ). Tanto al inicio de los tratamientos y a los 50 días de realizada la monta, se realizó un chequeo ecográfico transrectal con transductor linear (Mindray® DP 660 VET, 7,5 Mhz), para determinar el estado del útero y el diagnóstico de preñez respectivamente.

Para la calificación de la intensidad de la conducta estral, se consideraron los siguientes síntomas: chasquido de la boca, pestañeo vulvar y micción frecuente, vulva hinchada, cola alzada, inquietas y buscan al macho. Cuando las hembras presentaron el $100 \%$ de los síntomas antes señalados, el celo fue calificado como óptimo; con el 80 \% de sintomatología se calificó de muy bueno y con el 60 \% de bueno. Se consideró como inicio de presentación desde el momento de aplicación de la prostaglandina hasta el aparecimiento de los primeros síntomas que fueron el chasquido de la boca, inquietud, nuca estirada y orejas rectas y dirigidas hacia atrás. A cada una de las burras se le realizaron dos montas controladas al segundo y tercer día del estro, evaluando en este trabajo, el inicio y duración del celo manifiesto, la conducta e intensidad y la eficacia de los mismos a través del diagnóstico de preñez.

El análisis estadístico de las variables en estudio fue realizado mediante un ANOVA, en un diseño completamente al azar, considerando al tratamiento como un efecto fijo. Cuando la comparación entre los promedios de los tratamientos fue diferente estadísticamente $(\mathrm{p}<0,05)$, se aplicó el test de Duncan.

\section{RESULTADOS Y DISCUSIÓN}

La concentración media de $\mathrm{P} 4$ al inicio del ensayo fue de 9,1 $1 \pm, 6 \mathrm{ng} / \mathrm{mL}$ y a los 11 días de finalizados los protocolos alcanzaron un incremento promedio de $16,6 \pm 8,8 \mathrm{ng} / \mathrm{mL}$, al comparar este aumento en los tres tratamientos (figura 1), no se detectó diferencia entre ellos, permitiendo afirmar la eficacia de los mismos en cuanto a elevar los niveles de progesterona, desarrollar estructuras ováricas y provocar el reinicio de la funcionalidad ovárica. Contradiciendo estos resultados a lo manifestado por Handler et al. (2007), que en yeguas en anestro aplicó un tratamiento durante 11 días con dispositivos vaginales, obteniendo niveles de $\mathrm{P} 4$ al inicio y al final, similares a los de este estudio, pero señalando que en animales en anestro hay una reducida biosíntesis de GnRH y LH no siendo la P4 circulante capaz de estimular la síntesis de dichas hormonas y por tanto bastante improbable que los dispositivos induzcan a la presentación del celo y ovulación. 


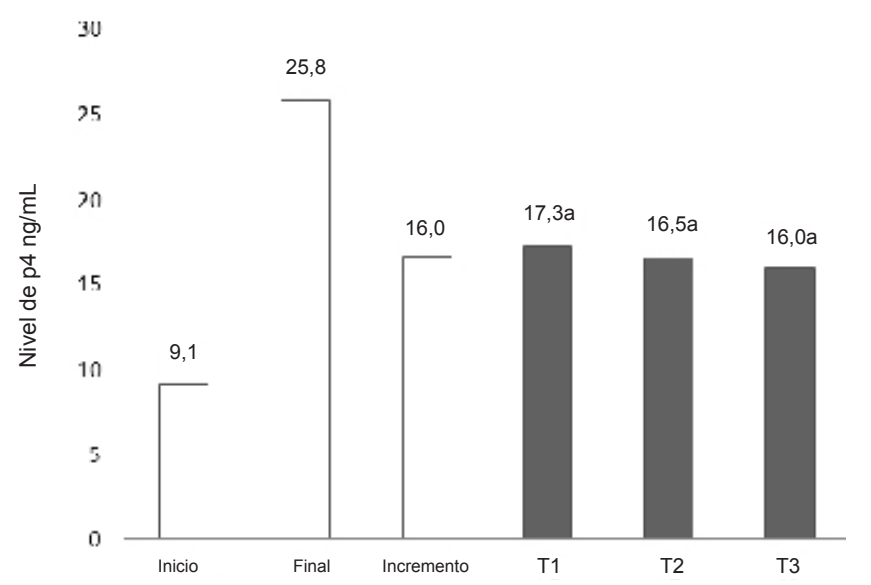

Promedio en la población

Incremento promedio en los tratamientos

Figura 1. Niveles de P4 sanguíneo en la población de burras y tratamientos investigados. (Levels of P4 in the blood on the population of jennies and investigated treatments).

La duración del celo en las burras Criollas del trópico seco, es similar a la encontrada por Blanchard et al. (1999), Taberner et al. (2008) y Galisteo y Perez-Marin (2010); todos ellos en burras de diferente raza con buena condición corporal y en zonas templadas, manifestando que la duración se ve afectada por factores como estación del año y ovulaciones múltiples; en el presente trabajo se observó que la duración se relacionó directamente con la intensidad de la conducta estral, pues aquellas burras que presentaron una óptima conducta tuvieron una duración de 4 y en las de menor intensidad de 5 días, esto posiblemente se deba a lo manifestado por Blanchard et al. (1999), de que las de mayor intensidad ovulan más pronto y por tanto terminan el celo ese mismo día o al otro día.

Todas las burras, en los tres protocolos, presentaron celo manifiesto, teniendo un similar tiempo de presentación de 3,3 $\pm 0,4$ días tanto las tratadas con progestágenos por vía auricular como vaginal, existiendo diferencia significativa $(\mathrm{p}<0,05)$ frente a las sincronizadas con la GnRH que presentaron celo a los 3,9 $9 \pm 0,5$ días de aplicada la PGF2 $\alpha$. Al respecto Blanchard et al. (1999), al aplicar sin saber el estado del ciclo sexual $5 \mathrm{mg}$ de PGF2 $\alpha$, consiguió el $76 \%$ de celos a los $4,4 \pm 1,6$ días y cuando aplicó 2 inyecciones de $10 \mathrm{mg}$ a intervalos de 16 días, tuvo una respuesta del $73 \%$ a los 4,5 días; Carluccio et al. (2008), en burras con buena condición corporal y ciclando consiguió 71,5\% de celos a los 3 días de la aplicación de 0,075 mg de Cloprostenol; Camillo et al. (2010), utilizó en un proceso de sincronización de donadoras y receptoras para TE, $3 \mathrm{mg}$ de PGF $2 \alpha$ con una muy buena respuesta sexual. Todo esto demuestra la buena fertilidad de la burra Criolla del trópico, con una respuesta positiva y temprana en todos los protocolos evaluados, también se puede apreciar que aún no se dispone en esta especie de un patrón de dosificación del luteolitico, estando de acuerdo con lo manifestado por Weber et al. (2001), de que el cuerpo lúteo de los equinos presenta una alta afinidad a la acción luteolitica de las prostaglandinas, requiriéndose de bajas dosis del mismo en relación a otras especies.
La sintomatología de celo observada en la burra Criolla es similar a la encontrada por Blanchard et al. (1999) y Taberner et al. (2008), en burras americanas (Equus asinus americanus) y catalanas respectivamente. Estos animales no manifestaron el síntoma del reflejo Flehemen y monta entre ellas que sí observaron Henry et al. (1998), en grupos de burras sincronizadas y al pastoreo libre, considerando que se dificultó la presentación de este síntoma por el pastoreo controlado que se lleva. En esta experiencia se registró el rebuzno como signo atípico. En este estudio el $46 \%$ de los protocolos tuvieron un óptimo celo, el $37 \%$ muy bueno y el $17 \%$ restante un celo bueno, destacando las burras del protocolo 1 que presentaron una mejor conducta estral (tabla I). Vale destacar que en el $100 \%$ de las burras a las que se aplicaron dispositivos vaginales, se apreció catarro vaginal notorio al momento de la extracción del dispositivo; sintomatología que también fue encontrada por Handler et al. (2007) y Miro et al. (2011), en el $100 \%$ de yeguas y burras respectivamente, sincro-nizadas con dispositivos, afectando notoriamente la concepción de dichos animales.

En la tabla I se presentan los resultados de preñez a los 50 días poscubrición $(\mathrm{p}<0,05)$, con el 100 y $75 \%$ de concepción alcanzados mediante el uso de implantes auriculares de progestágenos y de $\mathrm{GnRH}$, respectivamente, demostrando con ello que el uso de la GnRH como inductor de secreción de LH para el crecimiento de un CL dio excelente resultado en la tasa de preñez y no únicamente como ha venido siendo utilizada en la inducción de la ovulación (Handler et al., 1999; Carluccio et al., 2007). Los resultados en cuanto a la tasa de concepción con dispositivos vaginales fueron de $37,5 \%$, influyendo al parecer en esto la presencia del catarro vaginal que causó un ambiente inadecuado para el semen al momento de la monta directa, situación que no tendría ningún efecto si dicho proceso se hiciera mediante inseminación artificial. Es de destacar las hembras que no alcanzaron niveles de P4 mayores

Tabla I. Desempeño medio de las diferentes variables en los protocolos de sincronización evaluados (Mean performance of different variables in synchronization protocols evaluated).

\begin{tabular}{llll}
\hline Característica & T1 & T2 & T3 \\
\hline Conducta estral \% & & & \\
\hline Óptima & 38 & 50 & 50 \\
Muy buena & 62 & 25 & 25 \\
Buena & - & 25 & 25 \\
\hline
\end{tabular}

Nivel P4 al día $12^{*}$

\begin{tabular}{lcrl}
\hline Gestantes & $27,8 \pm 2,2$ & $30,7 \pm 0,6$ & $28,9 \pm 0,8$ \\
Vacías & - & $4,5 \pm 3,0$ & $23,8 \pm 12,0$
\end{tabular}

Celo manifiesto post sincronización (días)

\begin{tabular}{lccc}
\hline Óptima & $3,1 \pm 0,2$ & $3,8 \pm 0,4$ & $3,2 \pm 0,1$ \\
Muy buena & $3,4 \pm 0,5$ & $3,5 \pm 0,3$ & $3,2 \pm 0,2$ \\
Buena & - & $4,4 \pm 0,0$ & $3,9 \pm 0,8$ \\
\hline Concepción \% & $100^{\mathrm{a}}$ & $75^{\mathrm{b}}$ & $37,5^{\mathrm{c}}$
\end{tabular}

*Culminación protocolos ( $\mathrm{ng} / \mathrm{mL})$.

a,b,c Valores con distinta letra de superíndice, manifiestan diferencias significativas $(p<0,05)$. 
que $10 \mathrm{ng} / \mathrm{mL}$, fueron las que presentaron una conducta estral de menor intensidad más prolongada y no quedaron preñadas, sobre este aspecto Taberner $e t$ al. (2008), manifiestan que con una muy buena sintomatología estral en asociación con un tamaño y textura folicular adecuados, las probabilidades de ovulación dentro de las 24 horas siguientes son $>90 \%$, esto puede explicar porqué las burras que presentaron una conducta entre óptima y muy buena quedaron preñadas, exceptuando de este comportamiento a las hembras con el dispositivo vaginal.

\section{CONCLUSIONES}

La burra Criolla del trópico seco en el periodo del posparto y de amamantamiento que está ligado a la época de escasez de pasto, puede reiniciar su funcionalidad reproductiva con una nueva preñez empleando con éxito protocolos de sincronización, sea con implantes auriculares de progestágenos o con el uso de $\mathrm{GnRH}$, descartando el empleo en esta especie de dispositivos vaginales. Presenta un excelente comportamiento reproductivo en las condiciones ambientales desfavorables que se encuentran, aconsejando la cubrición sin monitoreo ecográfico folicular entre el segundo y cuarto día de iniciado el celo, representando una alternativa biotecnológica de fácil aplicación.

\section{AGRADECIMIENTOS}

A todos aquellos granjeros humildes del sector de Nambacola en la provincia de Loja-Ecuador, dueños de estos nobles animales que sin ningún interés ni egoísmo, colaboraron en el presente estudio.

\section{BIBLIOGRAFíA}

Blanchard, T.L.; Taylor, T.S. and Love, C.L. 1999. Estrus cycle characteristics and response to estrus synchronization in mammoth asses (Equus asinus americanus). Theriogenology, 52: 827-834.

Camillo, F.; Panzani, D.; Scollo, C.; Rota, A.; Crisci, A.; Vannozzi, I. and Balbo, S. 2010. Embryo recovery rate and recipiente's pregnancy rate after nonsurgical embryo transfer in donkeys. Theriogenology, 73: 959-965

Carluccio, A.; Panzani, S.; Tosi, U.; Faustini, M.; De Amicis, I. and Veronesi, M.C. 2007. Efficacy of $h C G$ and $G n R H$ for inducing ovulation in the Jenny. Theriogenology, 68: 914-919.
Carluccio, A.; Panzani, S.; Contri, A.; Tosi, U.; De Amicis, I. and Veronesi, M. 2008. Luteal function in jennies following PGF2 $\alpha$ treatment 3 days after ovulation. Theriogenology, 70: 121-125.

Dadarwal, D.; Tandon, S.N.; Purohit, G.N. and Pareek, P.K. 2004. Ultrasonographic evaluation of uterine involution and postpartum follicular dynamics of French Jennys (Equus asinus). Theriogenology, 62: 257-264.

Galisteo, J. and Perez-Marin, C.C. 2010. Factors affecting gestation length and estrus cycle cha-racteristics in Spanish donkey breeds reared in southern Spain. Theriogenology, 74: 443-450.

Handler, J.; Arbeiter, K. and Jöchle, W. 1999. Stimulation of fertile ovulations in mares during the transition period using an intravaginal progesterone device (CIDR-BTM) and subse-quently deslorelin acetate (OvuplantTM). Reprod Domest Anim, 34: 24.

Handler, J.; Schönlieb, S.; Hoppen, H.O. and Aurich, C. 2007. Influence of reproductive stage at PRIDTM insertion on synchronization of estrus and ovulation in mares. Anim Reprod Sci, 97: 382-393.

Henry, M.; Lody, L.D. and Gastal, M.M. 1998. Sexual behaviour of domesticated donkeys (Equus asinus) breeding under controlled or free range management systems. Appl Anim Behav Sci, 60: 263-276.

Jordana, J.; Ferrando, A.; Casa, M.; Loarca, A.; Martínez López, O.R.; Canelo, J.L.; Stemmer, A.; Aguirre, L.; Lara, M. y Álvarez, L.A. 2012. Diversidad y relaciones genéticas entre poblaciones asnales iberoamericanas. Actas Iberoamericanas de Conservación Animal AICA, 2: 207-213.

Lemma, A.; Schwarts, H.J. and Bekana, M. 2006. Aplication of ultrasonography in the study of reproductive system of tropical jennys (Equus asinus). Trop Anim Health Prod, 38: 267-274.

Meira, C.; Ferreira, J.C.; Papa, F.O.; Tornero, M.T. and Bicudo, S.D. 1995. Study of the estrus cycle in donkeys (Equus asinus) using ultrasonography and plasma progesterone concentrations. J Biol Reprod, 1: 403-410.

Miro, J.; Anglada, O.; Crisci, A.; Taberner, E. and Fabregas, N. 2010. Color doppler ultrasonographic evaluation of luteolysis in donkeys. Anim Reprod Sci, 45: 94.

Miro, J.; Torrens, M.; Giménez, M.; Roca, A.; Segura, F.; Fábregas, N. y Jordana, J. 2011 . Utilización de progestágenos por via vaginal (PRID®) para la inducción del celo en la burra de raza Balear. Memorias XI Simposio lberoamericano de Conservación de Recursos Zoogenéticos. João Pessoa. Brasil.

Pycock, J. 2001. Infertility in mares. In: Noakes, D.E.; Parkinson, J. England Editors. G.C.W. Arthur's veterinary reproduction and obstetrics. WB Saunders. Philadelphia.

Taberner, E.; Medrano, A.; Peña, A.; Rigau, T. and Miro, J. 2008. Oestrus cycle characteristics and prediction of ovulation in Catalonian jennies. Theriogenology, 70: 1489-1497.

Weber, J.A.; Causey, R.C. and Emmans, E.E. 2001. Induction of luteolysis in mares by ultrasound-guided intraluteal treatment with PGF2 $\alpha$. Theriogenology, 55: 1769-1776. 